\title{
The multitalented type III chaperones: all you can do with $15 \mathrm{kDa}$
}

\author{
Mario F. Feldman a, Guy R. Cornelis a,b,* \\ a Division of Molecular Microbiology, Biozentrum, University of Basel, Klingelbergstrasse 50-70, CH-4056 Basel, Switzerland \\ b Christian de Duve Institute of Cellular and Molecular Pathology and Faculté de Médecine, Université catholique de Louvain, Brussels, Belgium
}

Received 22 October 2002; received in revised form 7 January 2003; accepted 7 January 2003

First published online 30 January 2003

\begin{abstract}
Despite the fact that type III chaperones were discovered approximately 10 years ago, the precise role of most of them is still mysterious. A panoply of functions has been proposed for the members of this family of proteins. Type III chaperones have been suggested to act as anti-aggregation and stabilizing factors. They have also been proposed to keep their substrates in unfolded or partially folded structures, set a hierarchy on secretion, and participate in the regulation of the transcription of the type III substrates. Here, we review this enigmatic family of proteins, and discuss the experimental data supporting the roles proposed for type III chaperones.

(C) 2003 Federation of European Microbiological Societies. Published by Elsevier Science B.V. All rights reserved.
\end{abstract}

Keywords: Type III secretion; Chaperones; Bacterial pathogenesis; Yersinia; SycE

\section{Introduction}

The type III secretion (TTS) system is used by many pathogenic or symbiotic Gram-negative bacteria to inject proteins into the cytosol of host cells. TTS system is undoubtedly one of the most sophisticated devices that evolved in nature to allow translocation of proteins across lipid membranes. Such sophistication allows fast and efficient translocation of proteins through the bacterial inner membrane, the periplasm, the bacterial outer membrane, the LPS layer and, finally, the eukaryotic cell membrane. All these barriers seem to be crossed in a single step by the TTS pathway, delivering the proteins directly from the bacterial cytoplasm into the eukaryotic cell cytosol. Inside the eukaryotic cells, these proteins, called 'effectors', interfere with host cells signalling cascades. For example, the effectors can disrupt the cytoskeleton, cause apoptosis or modify the intercellular signalling, leading to the incapacitation of the host cell response. Proteins called 'translocators' are a second class of TTS substrates. These proteins form a pore in the eukaryotic cell membrane, allowing passage of the effectors. The machinery of secre-

\footnotetext{
* Corresponding author. Tel.: +41 (61) 2673111 ;

Fax: +41 (61) 2672118 .

E-mail address: guy.cornelis@unibas.ch (G.R. Cornelis).
}

tion is a complex organelle called 'injectisome', made of around 25 proteins. The achievement of the bacterial attack relies on the integration of several regulatory pathways controlling synthesis and assembly of the apparatus, and synthesis and secretion of the substrates. A central transcriptional activator usually belongs to the $\mathrm{AraC} /$ XylS family. The team is completed with an enigmatic family of proteins known as TTS chaperones. In this review we focus on the functions of this family of proteins. Several exhaustive reviews can be found for a comprehensive description of the TTS chaperones [1] and TTS systems in general [2-5].

\section{What is a TTS chaperone?}

Typical TTS chaperones are low molecular mass acidic proteins (ca $15 \mathrm{kDa}$ ) that specifically interact with only one or two effectors or translocators. Generally, the absence of the chaperone results in the lack of secretion of its cognate protein without altering the secretion of the other proteins. The TTS chaperones do not have ATP-binding domains and do not resemble at all the Hsp-related proteins. Usually, the chaperones act as dimers and bind to the $\mathrm{N}$-terminal region of the cognate protein. In general, the chaperones share little amino acid sequence homology between themselves, and the chaperone-binding domains of the different substrates do not present homology at all. 
It is almost a rule that the chaperones are encoded adjacently to their cognate effector. Interestingly, there are remarkable similarities between TTS chaperones and a group of chaperones that participate in flagellum assembly [6], which is not surprising since the flagellum is believed to be the ancestor of the TTS systems [7]. The archetype of the TTS chaperones is SycE from Yersinia enterocolitica that chaperones YopE (YerA in Yersinia pseudotuberculosis) [8]. Despite the fact that the crystal structure of SycE, and also the structure of SycE complexed with its binding domain on YopE, have been recently solved, the actual role of SycE is still not understood [9]. In the next sections, we review the present knowledge about this fastexpanding family of proteins, surveying the panoply of functions that has been proposed for the TTS chaperones. We focus especially on the analysis of the already demonstrated or suggested functions. Most of the examples come from research made in Yersinia spp., since the chaperones that participate in the Ysc TTS system of Yersinia have been studied intensively, but in some instances we need to refer to chaperones described in other well-studied TTS systems, such as those found in Salmonella spp., Shigella spp. and enteropathogenic Escherichia coli (EPEC).

\section{TTS chaperones as anti-aggregation and stabilizing factors}

In 1994, Menard et al. [10] demonstrated that in Shigella the translocators IpaB and IpaC, which together form a pore in the eukaryotic cell membrane, are independently associated in the bacterial cytoplasm with the chaperone IpgC. In addition, they showed that IpgC stabilizes IpaB and $\mathrm{IpaC}$ and prevents their premature association in the cytoplasm of Shigella cells. Therefore, the role of IpgC might be to prevent intrabacterial association of proteins that must interact outside of the bacterial cell.

A similar role was proposed for SycD, the chaperone of YopB and YopD, the translocators of the Ysc system of $Y$. enterocolitica [11]. However, in this case, binding between YopB and YopD occurred even in the presence of SycD. The authors showed that binding of SycD reduces the toxicity of GST-YopB hybrid in E. coli, and in addition they proposed that $\mathrm{SycD}$ prevents a premature association between YopB-YopD and another protein, LcrV, which is also required for pore formation [12].

It has been known for several years that, in the absence of SycE, YopE is unstable and rapidly degrades [13]. Indeed, the lack of stability of an effector in the absence of its chaperone is one of the hallmarks of the TTS chaperones. The reasons why SycE is necessary to stabilize YopE are unclear. Two recent reports suggest that SycE is necessary to mask an aggregation-prone region between amino acids 50-77 of YopE [14,15], since the presence of this domain creates the requirement of SycE for YopE secretion and stabilization. Intriguingly, the role of this region is unknown, given that YopE lacking this segment is still catalytically active [16].

The components of the machinery of secretion, the intracellular Yersinia Ysc proteins, were recently shown to bind to members of the TTS chaperones. Although the reasons why these proteins require chaperones are unknown, it was suggested that chaperone binding to a Ysc protein might prevent its premature interaction with other components of the secretion apparatus [17].

Thus it seems that some TTS chaperones are necessary to mask domains on the TTS substrates that are required for their functions or interactions and that could otherwise interfere with the process of translocation, cause aggregation, or be toxic for the bacterial cells (Fig. 1).

\section{TTSS chaperones as signals for secretion and hierarchy-determining factors}

The first 15 amino acids of YopE are sufficient for secretion of several hybrid proteins. However, Cheng and Schneewind [18] identified a second signal located at residues 15-100 of YopE that is only recognized by the TTS system when SycE is present and improves the targeting of the protein to the injectisome. This finding provided experimental support to the early suggestion that the chaperones could act as secretion pilots [8]. More recently, Birtalan et al. [9] crystallized the N-terminal region of YopE complexed with SycE. The described structure resembles the one found for the complex between the $\mathrm{N}$-terminal region of the Salmonella effector Spt complexed with its chaperone SicP [19]. Since neither the binding domain nor the chaperones have appreciable sequence similarity, Birtalan et al. suggested that these complexes could function as general, three-dimensional TTS signals [9].

In Yersinia, only three of the effectors (YopE, YopH and YopT) have a chaperone, and so far there is no chaperone identified that specifically assists secretion of the remaining effectors (YopM, YopP/YopJ, YopO/YpkA). Suggestively, YopE, YopH and YopT share the function of protecting Yersinia from phagocytosis by interfering with cytoskeleton dynamics. Since a macrophage is able to phagocytose a bacterium in less than a minute, the secretion of the antiphagocytic Yops should be a priority for Yersinia to survive extracellularly inside the host. Accordingly, it has been shown that YopH is delivered into macrophages within a minute of infection [20]. The fact that the three Yops that are served by a chaperone have an antiphagocytic role led Birtalan et al. to suggest the attractive hypothesis that the three-dimensional signals described above, formed by the chaperones binding to their substrates, may be responsible for setting a temporal hierarchy on secretion (Fig. 2). However, to date, no solid experimental data supporting this theory is available.

Nevertheless, there is some evidence of a quantitative 
hierarchy on secretion. Boyd et al. [14] showed that a hybrid comprising the first 15 amino acids of YopE fused to adenylate cyclase (Cya) was poorly translocated by wild-type bacteria. On the contrary, high levels of the hybrid protein were injected by a multimutant Yersinia strain devoid of most of the TTS substrates, suggesting the existence of competition between the Yops for delivery into eukaryotic cells. However, if the fusion protein contained 130 amino acids of YopE, which means a functional SycE binding domain, the fusion YopE-Cya was injected in high levels by both wild-type and polymutant strains. Based on these observations, Boyd et al. hypothesized that the binding of the chaperones confers to the effector a privilege in secretion. Recently, $\mathrm{SycH}$ has been also implicated in the imposition of such a hierarchy of secretion by the group of S. Straley [21].

Undoubtedly, the elucidation of the first steps in substrate recognition and engagement into the injectisomes will help to understand how the chaperones work as signals and how is this putative hierarchy in secretion is established.

\section{TTS chaperones as antifolding factors}

It has been shown in Pseudomonas syringae that the effectors and translocators travel to the eukaryotic cell through the injectisomes [22,23]. Structural studies in Salmonella, Shigella and Yersinia suggest that the central conduit of the secretion channel is between 2 and $3 \mathrm{~nm} \mathrm{[24-}$ 26]. Therefore, if small proteins could still travel folded, the channels are too narrow to fit large proteins that are completely folded. The fact that the size of TTS substrates is between 15 and $80 \mathrm{kDa}$ or more leads to the question of how proteins unfold or are kept in an unfolded state competent with secretion.

In the study of Stebbins and Galan already mentioned [19], the structure of the complex between the chaperonebinding domain of Salmonella effector SptP and its cognate chaperone $\mathrm{SicP}$ revealed that this domain is maintained in an extended, unfolded conformation by means of the chaperone. Although the idea that chaperones keep their substrates in a secretion-competent state is as old as the discovery of the chaperones [8], the work of Stebbins and Galan gave the first experimental support to this idea. However, the role of TTS chaperones as antifolding factors has been challenged by Birtalan et al. [9], who showed that the YopE-SycE complex purified from E. coli is catalytically active. Furthermore, Luo et al. [27] presented biochemical evidence that in the complexes between the effector Tir from enterohemorrhagic E. coli (EHEC) and its chaperone CesT, as well as in the complex between the
A

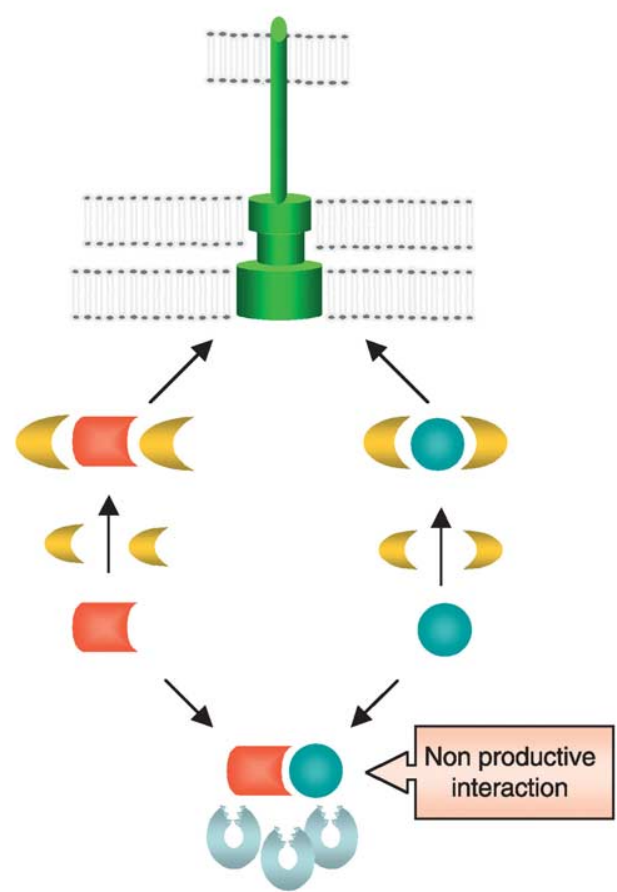

B
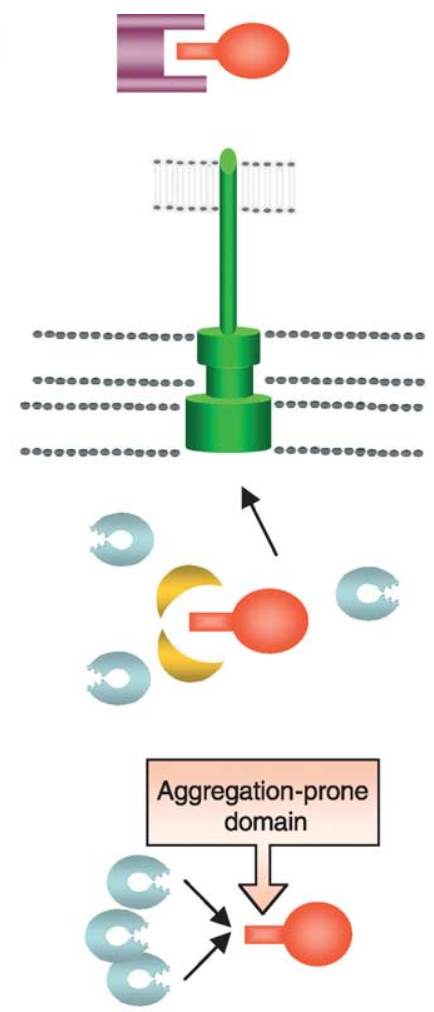

Fig. 1. TTS chaperones as anti-aggregation and stabilizing factors. A: Two TTS substrates interact out of the cell. A TTS chaperone can bind individually to both substrates, avoiding premature non-productive intracellular interactions that would lead to the degradation of the proteins. B: A TTS chaperone binds to an aggregation-prone domain in an effector. The binding of the chaperone to this domain (probably required for the action of this effector inside the eukaryotic cell) protects the protein from degradation. 


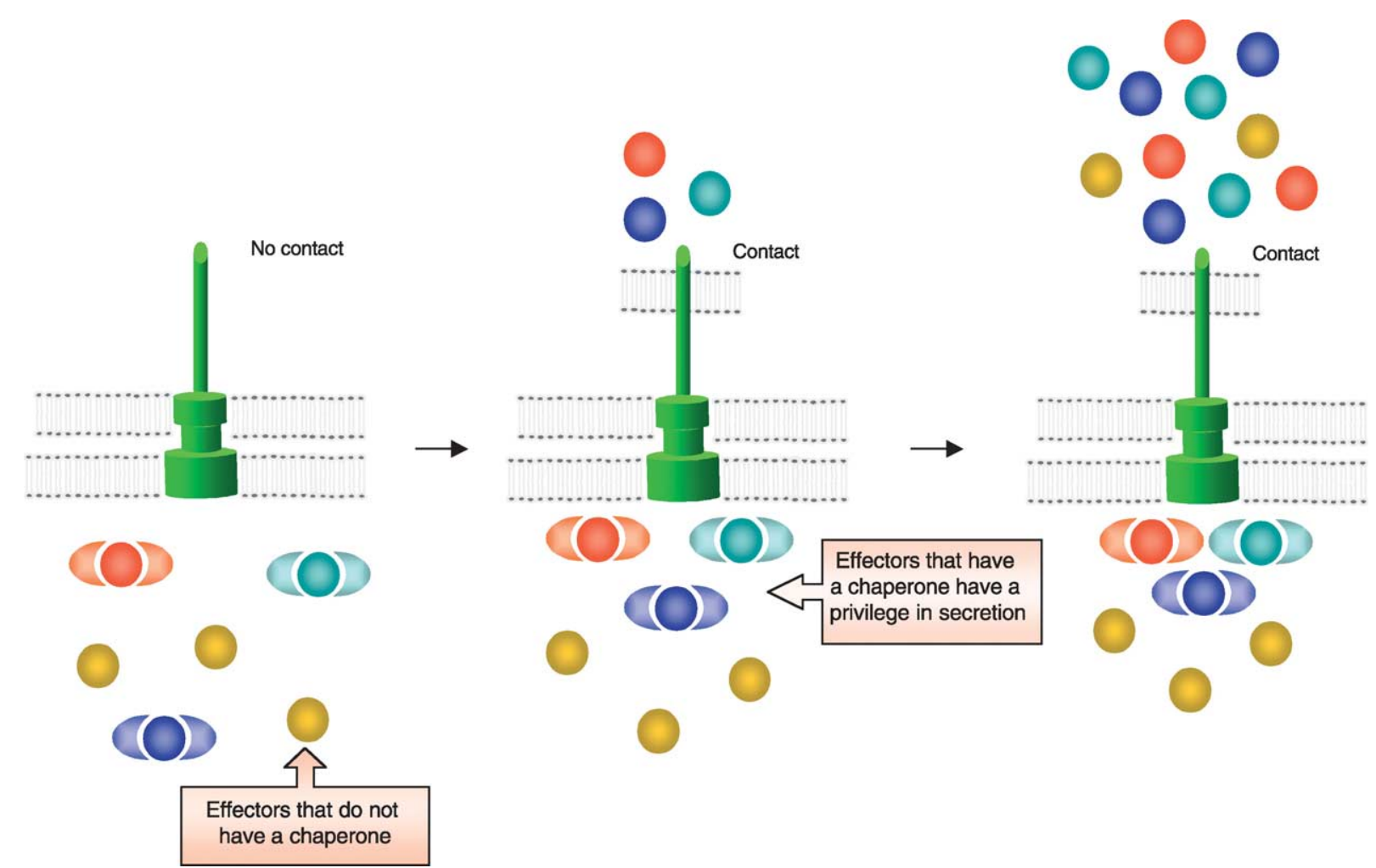

Fig. 2. TTSS chaperones as signals for secretion and hierarchy determining factors. TTS chaperones binding could create three-dimensional signals that could confer to their substrates temporal or quantitative privileges in secretion.

Salmonella effector SigD and its chaperone SigE, the effector proteins are not globally unfolded in the presence of their cognate chaperones. However, it cannot be excluded that in vivo the situation is different. The secretion process is extremely fast and it is quite possible that the complex is recognized immediately by other proteins of the TTS system, which might stabilize the complex in an unfolded conformation (Fig. 3). In our recent studies of secretion of YopE-DHFR hybrids in vivo, we showed that SycE prevents folding of the DHFR moiety of the hybrid protein, allowing its secretion, and we proposed that the binding of SycE to YopE in vivo is cotranslational [15]. This putative role for the chaperones is analogous to the role of $\mathrm{SecB}$, which participates in secretion by the Sec system [28].

\section{TTS chaperones as regulators of expression}

When YerA (SycE in Y. enterocolitica) was discovered in Y. pseudotuberculosis it was thought to be the transcriptional regulator of YopE (in fact, YerA stands for $Y o p E$ regulating protein $A$ )[29]. When it was shown that SycE binds to YopE and stabilizes the protein in the cytoplasm, it was suggested to be a chaperone rather than a regulator and it was called SycE (for specific $Y \mathrm{op} E$ chaperone) [8]. Now, it seems that some TTS chaperones are involved in the regulation of the expression of some components of the TTS system.

The first demonstration of a TTS chaperone involved in regulation of virulence genes comes from the work of Darwin and Miller [30], who showed that the Salmonella chaperone SicA can interact with InvF, a transcriptional activator of the AraC/XylS family. The complex SicAInvF is required to activate the transcription of a subset of genes involved in the TTS systems, including many effectors. One common property of the InvF/SicA-regulated operons is that they are all required for bacterial invasion of eukaryotic cells. A similar role has been shown for the chaperone LcrH (the Y. pseudotuberculosis homo$\log$ of SycD, the chaperone of YopB and YopD in Y. enterocolitica). A $l c r H$ null mutant constitutively produces Yops, and therefore LcrH is important in establishing a negative feedback regulation of the proteins secreted by the TTS system [31].

$\mathrm{SycH}$, the chaperone of $\mathrm{YopH}$, seems also to be involved in the regulation of the type III pathway. A $\mathrm{SycH}$ mutant expresses and secretes significantly less Yops than wild-type cells and overexpression of $\mathrm{SycH}$ in $Y$. enterocolitica causes a general increase in the synthesis and secretion of TTS substrates [32]. Recently, it was also shown that in Shigella the chaperone IpgC acts as a coactivator that allows the transcription factor MxiE to activate transcription at its target promoters [33]. 


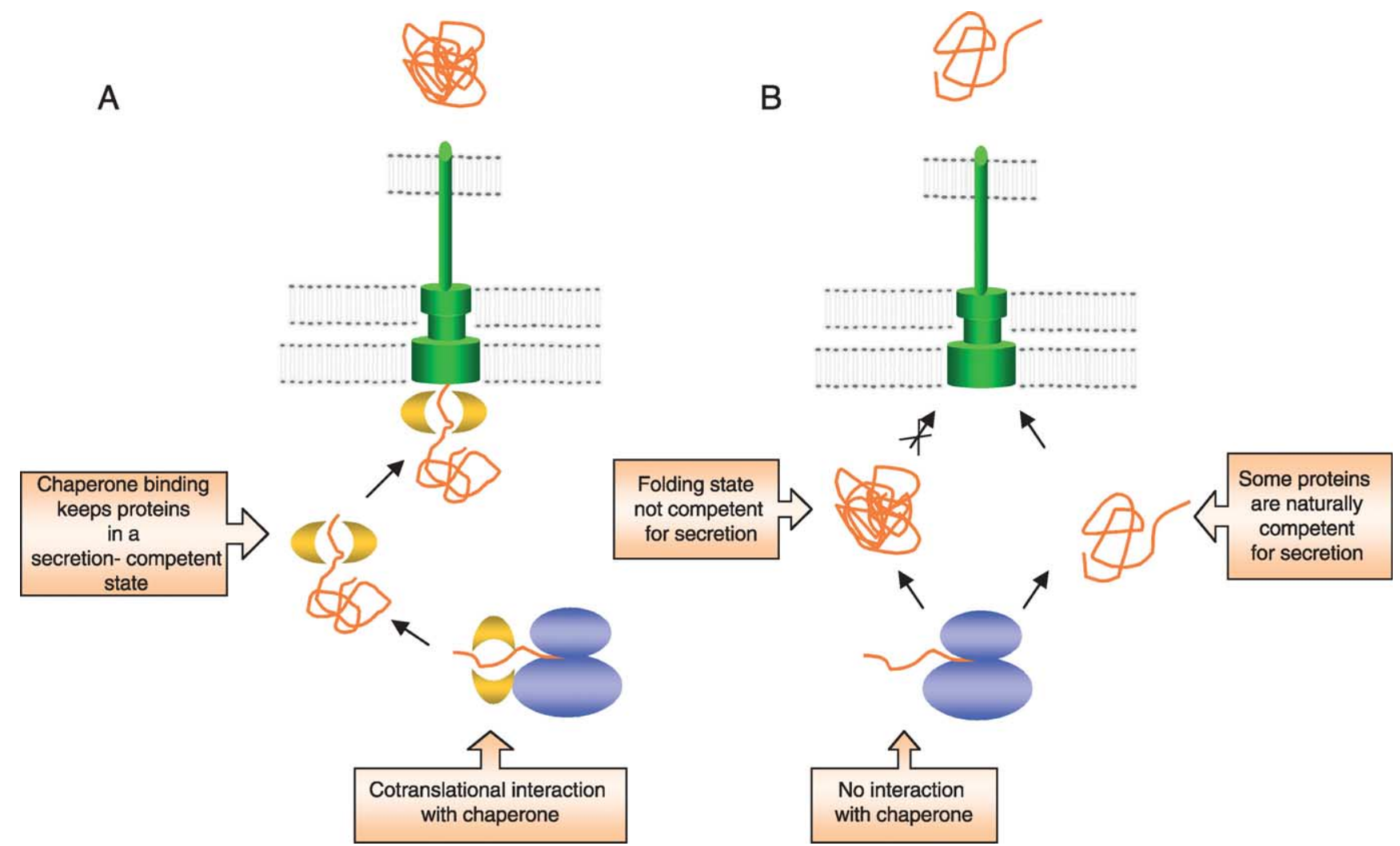

Fig. 3. TTS chaperones as antifolding factors. A: A chaperone binds to its cognate protein in a cotranslational way, preventing its folding. The chaperone keeps the protein in a secretion-competent conformation, compatible with secretion. B: Some TTS substrates do not need a chaperone and are naturally competent for secretion.

Feedback inhibition of transcription of the TTS substrates is known to occur when the channels are closed. The expression of most of the genes of the TTSS is known to be activated upon contact with a eukaryotic cell or in conditions mimicking such contact in vitro. It seems that TTS chaperones act as sensors of the intracellular levels of their cognate proteins. Upon contact with the eukaryotic cells, secretion of the effectors and translocators starts, the intracellular amount of free chaperone increases, allowing them to activate or inhibit the transcriptional regulators (Fig. 4). For a more detailed description about regulation of type III secretion we suggest the recent review by Francis et al. [34].

\section{Conclusions and perspectives}

Table 1 summarizes the functions proposed for several TTS chaperones. Due to space restrictions, in the text we have only discussed just some examples for each role. Some of the roles proposed for the members of the family can be considered well established; meanwhile, some of the functions proposed are still hypothetical. Most of the studies were carried out analyzing in vitro secretion of the effectors and translocators instead of translocation into eukaryotic cells. During infection, contact with eukaryotic cells triggers the synthesis and translocation of the effectors. Calcium chelation and Congo red treatment mimic the cell contact for Yersinia and Shigella, respectively, but the mechanism is still not understood. These treatments have shown to be a wonderful tool for studying the TTSS, since they lead to unmasking these sophisticated weapons present in many pathogens. However, the more optimistic estimations of the rate of secretion of the TTS substrates show that less than 5\% of the effectors produced is indeed secreted in in vitro secretion conditions. Obviously, this must not be the case in a real infection, for otherwise all this sophistication would be hard to understand. In addition, many of the experiments in the early times were carried out in high-copy number plasmids. With the present knowledge about TTS system regulation, it is clear that some of these results must be taken with caution and some of them need to be reconfirmed.

Often there is a tendency to extend a role described for one chaperone to another one or to all the members of the family. It is highly probable that chaperones evolved from a common ancestor, but they specialized to accomplish different roles during secretion. It is possible that some chaperones have more than one role and that not all the 
Table 1

\begin{tabular}{|c|c|c|}
\hline Proposed function & Examples & References \\
\hline Anti-aggregation, stabilization & SycE, IpgC, IpgE, SycN, YscB, SycD, Spa15, SicA, YscG & {$[8,10,11,17,36-38]$} \\
\hline Maintenance of a secretion-competent state, antifolding & SycE, SicP, Spa15 & {$[15,19,36]$} \\
\hline Secretion pilot & YopE, SicP & {$[9,18]$} \\
\hline Hierarchy of secretion & SycE, SycH, SicP & {$[14,9,21]$} \\
\hline Regulation of TTS system & $\mathrm{SycH}, \mathrm{SicA}, \mathrm{IpgC}, \mathrm{LcrH}$ & {$[30-33]$} \\
\hline
\end{tabular}

chaperones share the same functions. However, we have to admit that, despite the fact that TTS chaperones were discovered approximately 10 years ago, the precise role of most of them is unknown. Structural biologists have shown crystal structures of several chaperones, structures of the catalytic domains of many effectors and even the structures of two chaperones bound to their respective binding domains in the effectors $[9,16,35]$. However, these studies, although highly valuable, did not give a conclusive answer as to the function of type III chaperones. Curiously, so far not a single full-length effector bound to its chaperone could be crystallized. But, undoubtedly, to understand how chaperones act, the elucidation of the early steps in the secretion is needed. Many important questions are waiting for answers: how are the TTS substrates recognized by the machine? Which proteins interact with the chaperones? How are the chaperones detached from their substrates? Is there a temporal chaperone-mediated hierarchy in the secretion of the effectors?

Further work will clarify the actual roles of the TTS chaperones, and this clarification will contribute to the understanding of the TTS pathway itself. It may also in the future contribute to the development of new pharma-

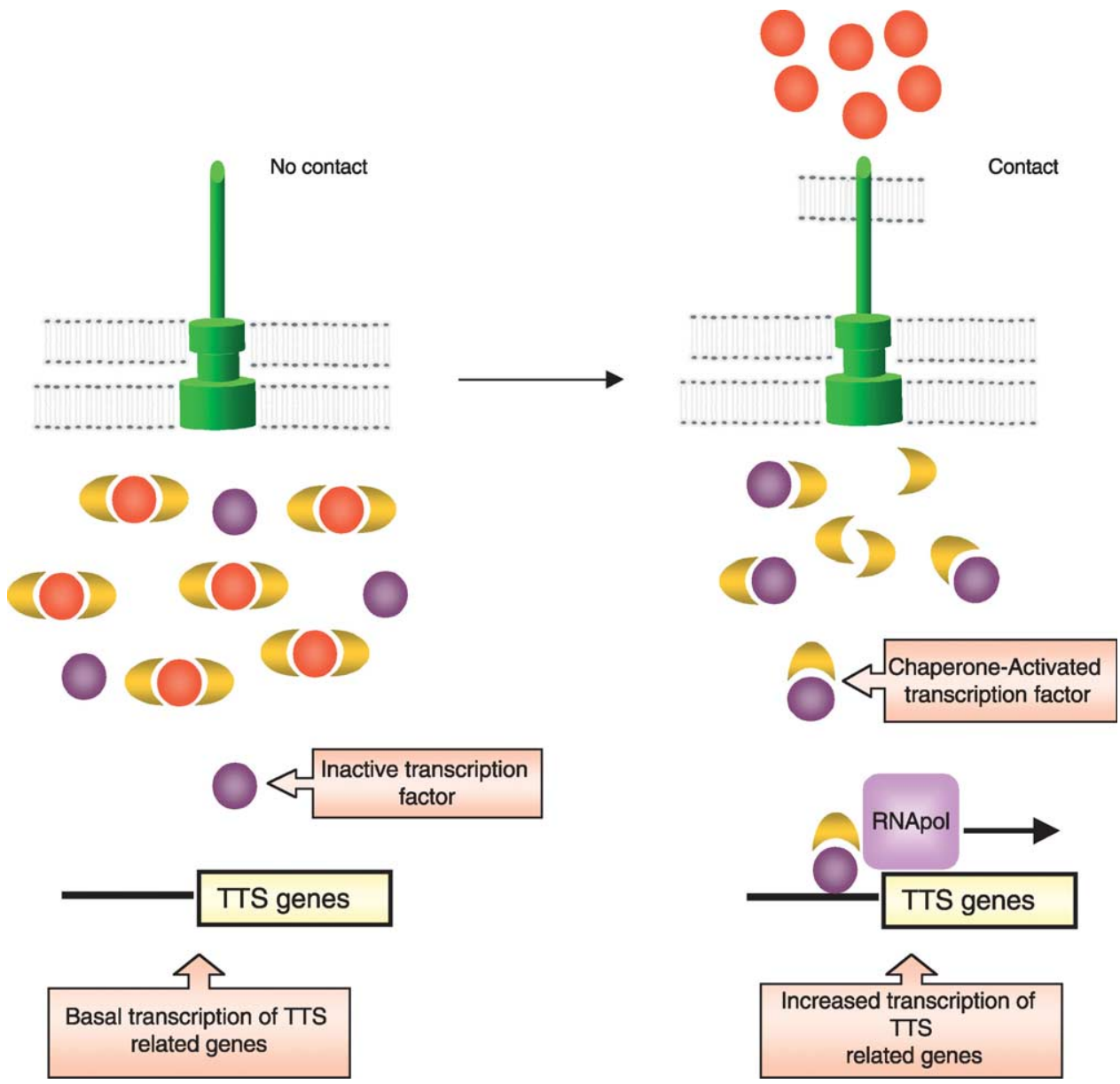

Fig. 4. TTS chaperones as regulators of expression. In the absence of contact with a eukaryotic cell, the chaperones are bound to their cognate proteins, and transcription of the TTS-related genes is kept at a basal level. When a contact is made between the bacteria and the eukaryotic cell, the substrates are translocated into the eukaryotic cell and the concentration of the free chaperones in the bacterial cytoplasm increases. Some chaperones can then activate a transcription factor, increasing the transcription level. 
cological agents interfering with this important virulence mechanism.

\section{Acknowledgements}

We thank Jaime Mota and Paul Troisfontaines for the critical reading of the manuscript and Trui Denecker for the help in the design of the figures. The laboratory is supported by the Swiss National Science Foundation (Grant 32-65393.01), the Belgian 'Fonds National de la Recherche Scientifique Médicale’ (Convention 3.4595.97), the Belgian 'Direction générale de la Recherche Scientifique Communauté Française de Belgique' (Action de Recherche Concertée 94/99-172). The laboratory also participates in the EU TMR network 'MEMBMACS' (contract HRPN-CT-2000-00075).

\section{References}

[1] Page, A.L. and Parsot, C. (2002) Chaperones of the type III secretion pathway: jacks of all trades. Mol. Microbiol. 46, 1-11.

[2] Cornelis, G.R. (2002) Yersinia type III secretion: send in the effectors. J. Cell Biol. 158, 401-408.

[3] Cornelis, G.R. and Van Gijsegem, F. (2000) Assembly and function of type III secretory systems. Annu. Rev. Microbiol. 54, 735774.

[4] Galan, J.E. (2001) Salmonella interactions with host cells: type III secretion at work. Annu. Rev. Cell Dev. Biol. 17, 53-86.

[5] Buttner, D. and Bonas, U. (2002) Port of entry - the type III secretion translocon. Trends Microbiol. 10, 186-192.

[6] Bennett, J.C. and Hughes, C. (2000) From flagellum assembly to virulence: the extended family of type III export chaperones. Trends Microbiol. 8, 202-204.

[7] Aizawa, S.I. (2001) Bacterial flagella and type III secretion systems. FEMS Microbiol. Lett. 21, 157-164.

[8] Wattiau, P. and Cornelis, G.R. (1993) SycE, a chaperone-like protein of Yersinia enterocolitica involved in the secretion of YopE. Mol. Microbiol. 8, 123-131.

[9] Birtalan, S.C., Phillips, R.M. and Ghosh, P. (2002) Three-dimensional secretion signals in chaperone-effector complexes of bacterial pathogens. Mol. Cell 9, 971-980.

[10] Menard, R., Sansonetti, P., Parsot, C. and Vasselon, T. (1994) Extracellular association and cytoplasmic partitioning of the IpaB and IpaC invasins of S. flexneri. Cell 79, 515-525.

[11] Neyt, C. and Cornelis, G.R. (1999) Role of SycD, the chaperone of the Yersinia Yop translocators YopB and YopD. Mol. Microbiol. 31, $143-156$.

[12] Holmstrom, A., Olsson, J., Cherepanov, P., Maier, E., Nordfelth, R., Pettersson, J., Benz, R., Wolf-Watz, H. and Forsberg, A. (2001) LcrV is a channel size-determining component of the Yop effector translocon of Yersinia. Mol. Microbiol. 39, 620-632.

[13] Frithz-Lindsten, E., Rosqvist, R., Johansson, L. and Forsberg, A. (1995) The chaperone-like protein YerA of Yersinia pseudotuberculosis stabilizes YopE in the cytoplasm but is dispensible for targeting to the secretion loci. Mol. Microbiol. 16, 635-647.

[14] Boyd, A.P., Lambermont, I. and Cornelis, G.R. (2000) Competition between the Yops of Yersinia enterocolitica for delivery into eukaryotic cells: role of the SycE chaperone binding domain of YopE. J. Bacteriol. 182, 4811-4821.

[15] Feldman, M.F., Müller, S., Wüest, E. and Cornelis, G.R. (2002)
SycE allows secretion of YopE-DHFR hybrids by the Yersinia enterocolitica type III Ysc system. Mol. Microbiol. 46, 11831197.

[16] Evdokimov, A.G., Tropea, J.E., Routzahn, K.M. and Waugh, D.S. (2002) Crystal structure of the Yersinia pestis GTPase activator YopE. Protein Sci. 11, 401-408.

[17] Day, J.B., Guller, I. and Plano, G.V. (2000) Yersinia pestis YscG protein is a Syc-like chaperone that directly binds yscE. Infect. Immun. 68, 6466-6471.

[18] Cheng, L.W., Anderson, D.M. and Schneewind, O. (1997) Two independent type III secretion mechanisms for YopE in Yersinia enterocolitica. Mol. Microbiol. 24, 757-765.

[19] Stebbins, C.E. and Galan, J.E. (2001) Maintenance of an unfolded polypeptide by a cognate chaperone in bacterial type III secretion. Nature 414, 77-81.

[20] Andersson, K., Magnusson, K.E., Majeed, M., Stendahl, O. and Fallman, M. (1999) Yersinia pseudotuberculosis-induced calcium signaling in neutrophils is blocked by the virulence effector YopH. Infect. Immun. 67, 2567-2574.

[21] Wulff-Strobel, C.R., Williams, A.W. and Straley, S.C. (2002) LcrQ and $\mathrm{SycH}$ function together at the Ysc type III secretion system in Yersinia pestis to impose a hierarchy of secretion. Mol. Microbiol. 43, 411-423.

[22] Li, C.M., Brown, I., Mansfield, J., Stevens, C., Boureau, T., Romantschuk, M. and Taira, S. (2002) The Hrp pilus of Pseudomonas syringae elongates from its tip and acts as a conduit for translocation of the effector protein HrpZ. EMBO J. 21, 1909-1915.

[23] Jin, Q. and He, S.Y. (2001) Role of the Hrp pilus in type III protein secretion in Pseudomonas syringae. Science 294, 2556-2558.

[24] Kubori, T., Matsushima, Y., Nakamura, D., Uralil, J., Lara-Tejero, M., Sukhan, A., Galan, J.E. and Aizawa, S.I. (1998) Supramolecular structure of the Salmonella typhimurium type III protein secretion system. Science 280, 602-605.

[25] Blocker, A., Jouihri, N., Larquet, E., Gounon, P., Ebel, F., Parsot, C., Sansonetti, P. and Allaoui, A. (2001) Structure and composition of the Shigella flexneri 'needle complex', a part of its type III secretion. Mol. Microbiol. 39, 652-663.

[26] Hoiczyk, E. and Blobel, G. (2001) Polymerization of a single protein of the pathogen Yersinia enterocolitica into needles punctures eukaryotic cells. Proc. Natl. Acad. Sci. USA 98, 4669-4674.

[27] Luo, Y., Bertero, M.G., Frey, E.A., Pfuetzner, R.A., Wenk, M.R., Creagh, L., Marcus, S.L., Lim, D., Sicheri, F., Kay, C., Haynes, C., Finlay, B.B. and Strynadka, N.C. (2001) Structural and biochemical characterization of the type III secretion chaperones CesT and SigE. Nat. Struct. Biol. 29, 29.

[28] Driessen, A.J. (2001) SecB, a molecular chaperone with two faces. Trends Microbiol. 9, 193-196.

[29] Forsberg, A. and Wolf-Watz, H. (1990) Genetic analysis of the yopE region of Yersinia spp.: identification of a novel conserved locus, yerA, regulating yopE expression. J. Bacteriol. 172, 15471555.

[30] Darwin, K.H. and Miller, V.L. (2001) Type III secretion chaperonedependent regulation: activation of virulence genes by SicA and InvF in Salmonella typhimurium. EMBO J. 20, 1850-1862.

[31] Francis, M.S., Lloyd, S.A. and Wolf-Watz, H. (2001) The type III secretion chaperone LcrH cooperates with YopD to establish a negative, regulatory loop for control of Yop synthesis in Yersinia pseudotuberculosis. Mol. Microbiol. 42, 1075-1093.

[32] Cambronne, E.D., Cheng, L.W. and Schneewind, O. (2000) LcrQ/ YscM1, regulators of the Yersinia yop virulon, are injected into host cells by a chaperone-dependent mechanism. Mol. Microbiol. 37, 263-273.

[33] Mavris, M., Page, A.L., Tournebize, R., Demers, B., Sansonetti, P. and Parsot, C. (2002) Regulation of transcription by the activity of the Shigella flexneri type III secretion apparatus. Mol. Microbiol. 43, 1543-1553.

[34] Francis, M.S., Wolf-Watz, H. and Forsberg, A. (2002) Regulation 
of type III secretion systems. Curr. Opin. Microbiol. 5, 166172.

[35] Birtalan, S. and Ghosh, P. (2001) Structure of the Yersinia type III secretory system chaperone SycE. Nat. Struct. Biol. 8, 974-978.

[36] Page, A.L., Sansonetti, P. and Parsot, C. (2002) Spa15 of Shigella flexneri, a third type of chaperone in the type III secretion pathway. Mol. Microbiol. 43, 1533-1542.
[37] Niebuhr, K., Jouihri, N., Allaoui, A., Gounon, P., Sansonetti, P.J. and Parsot, C. (2000) IpgD, a protein secreted by the type III secretion machinery of Shigella flexneri, is chaperoned by IpgE and implicated in entry focus formation. Mol. Microbiol. 38, 8-19.

[38] Tucker, S.C. and Galan, J.E. (2000) Complex function for SicA, a Salmonella enterica serovar typhimurium type III secretion-associated chaperone. J. Bacteriol. 182, 2262-2268. 